\title{
A Comparison between The Incidence of Paresthesia in Median and Paramedian Approaches of Intrathecal Anesthesia in Parturients Undergoing Cesarean Sections: A Randomized Controlled Trial
}

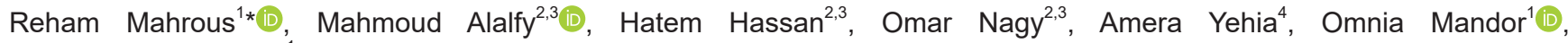 \\ Amr K. Abdel Hakeem \\ ${ }^{1}$ Department of Anesthesia, Surgical ICU, and Pain Management, Cairo University, Giza, Egypt; ${ }^{2}$ Department of Reproductive \\ Health and Family Planning, National Research Centre, Dokki, Egypt; ${ }^{3}$ Department of Obstetrics and Gynecology, Aljazeerah \\ Hospital, Giza, Egypt; ${ }^{4}$ Department of Obstetrics and Gynecological Diseases, Cairo University, Giza, Egypt
}

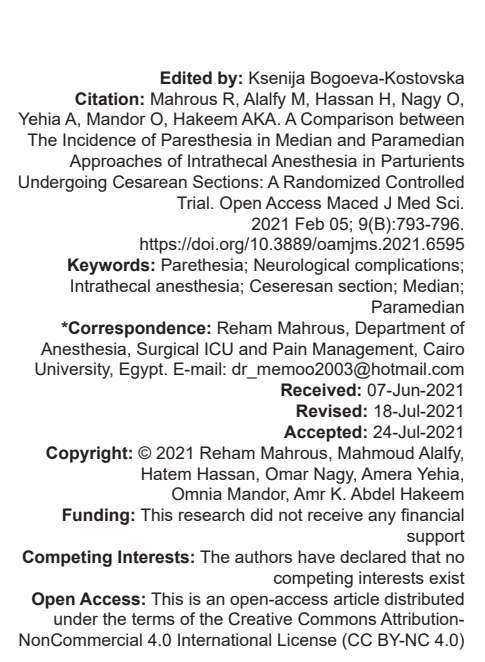

Abstract

BACKGROUND: Paresthesia with intrathecal anesthesia occurs when the entry of the needle causes an uncomfortable pain, burning, or electric sensation that usually radiates to the buttocks or legs. The importance of avoiding paresthesia is to lower the risk of postoperative neurological problems, in addition to reducing the incidence of that unpleasant sensation. The majority of reported occurrences of nerve injury caused by spinal anesthesia were preceded by paresthesia during the spinal anesthesia needle insertion. To the best of our knowledge, no studies have been done to compare the incidence of paresthesia in cesarean sections using median and paramedian routes to provide spinal anesthetic.

AIM: Our study aimed to compare the incidence of paresthesia in the median and paramedian approaches of intrathecal anesthesia to predict its association with nerve injury.

METHODOLOGY: Two hundred-ninety-six parturients scheduled for elective cesarean sections under spinal anesthesia were in the study from November 2020 to January 2021. They were divided into two groups. The median group $(n=157)$ and the paramedian group $(n=135)$ according to the approach used for providing spinal anesthesia. The incidence of paresthesia was compared between both groups. The number of trials in each approach and the occurrence of postoperative neurological complications (for example: nerve injury, paraplegia, or foot drop) were also documented.

RESULTS: The sample size was calculated based on a pilot study that was conducted before the original study. The calculated sample size was based on an alpha error of 0.05 and $90 \%$ power. The incidence of paresthesia was higher in the median group $(10.7 \%)$ than the paramedian (3.7) group with statistical significance $(p=0.039)$. In both groups no postoperative neurological problems were noted in any groups.

CONCLUSION: The median group has significantly more incidence of paresthesia than the paramedian group. The significance of this finding is that the paramedian approach is expected to be less likely to cause neurologic problems during spinal anesthesia.

\section{Introduction}

The number of cesarean sections worldwide has markedly been increasing [1], [2]. Intrathecal anesthesia is preferred by most obstetric anesthesiologists over general anesthesia as they offer good efficacy with a higher safety profile for both the mother and the fetus [3].

Spinal anesthesia is conducted through one of two approaches, the median or paramedian approach. The median approach is the most used technique. However, it may be technically difficult to perform in elderly patients (as they have calcified interspinous ligaments), in obese patients, and sometimes in parturient (due to difficulties in positioning and exaggerated lumbar lordosis) [4].

Complications with spinal anesthesia are not common and one of the most serious complications is nerve injury [5].
Paresthesia in general is defined as abnormal burning or tingling that is a common symptom of Multiple sclerosis disease [6]. Paresthesia during intrathecal anesthesia is defined as an unpleasant pain, burning, or electric sensation with the introduction of the needle that usually radiates to the buttocks or legs [7].

The incidence of paresthesia during performing neuro-axial anesthesia is up to $56 \%$ in some studies [8].

The importance of minimizing the incidence of paresthesia besides decreasing the incidence of that unpleasant sensation is to reduce the risk of postoperative neurological complications. Most of the reported cases of nerve injury with spinal anesthesia were preceded by paresthesia during the introduction of the spinal anesthesia needle [9].

Several studies were done to assess the association of different factors as the size of the needle or the use of an introducer with the incidence of paresthesia [7]. The difference in the incidence of 
paresthesia between using median and Paramedian approaches for spinal anesthesia is not studied yet [7].

Our study aimed to compare the incidence of paresthesia in the median and paramedian approaches of intrathecal anesthesia to predict its association with nerve injury.

\section{Patients and Methods}

\section{Study population}

This study is a randomized controlled trial carried out at Al Jazeera Hospital from November 2020 till January 2021. Three hundred and fifteen parturients were assessed for eligibility criteria and 296 were found eligible and recruited and divided into two groups. The parturients were randomly allocated to the two groups using an online random number generator. The median approach of intrathecal anesthesia was used in the first group (157 cases) and the para-median approach was used in the second one (139 cases). The random sequence was placed in opaque envelopes with serial numbers.

The study was approved by the Ethical Committee of Al Gazeera Hospital and was done with strict adherence to the tenets of the Declaration of Helsinki. The procedures were explained in simple words for all the participants, then written informed consent was obtained. Our inclusion criteria were: Age 18-40 years, parturients scheduled for elective cesarean section, and with American Society of Anesthesiologists (ASA) score of II. The exclusion criteria were: Age $<18$ years or $>40$ years, ASA score>II, emergency cesarean section, or the presence of contraindications for spinal anesthesia.

Twelve out of the 296 cases (eight in the median group and four in the paramedian one) were excluded as they required $>3$ trials to induce spinal anesthesia and the original approach was aborted (Figure 1).

\section{Anesthesia technique}

After application of the standard monitoring (electrocardiogram, non-invasive blood pressure monitor, and pulse oximetry), insertion of an $18 \mathrm{G}$ peripheral intravenous line, and giving $10 \mathrm{ml} / \mathrm{kg}$ of lactated ringer as a preload, intrathecal anesthesia was given.

The median approach for intrathecal anesthesia was used in the first group, and the paramedian approach was used in the second group.

Senior anesthesiologists (with a minimum of 5 years experience in obstetric anesthesia) conducted spinal anesthesia in the sitting position at the level of the lumbar intervertebral space L4-5. The midline approach was performed using standard technique; the paramedian approach was performed by inserting

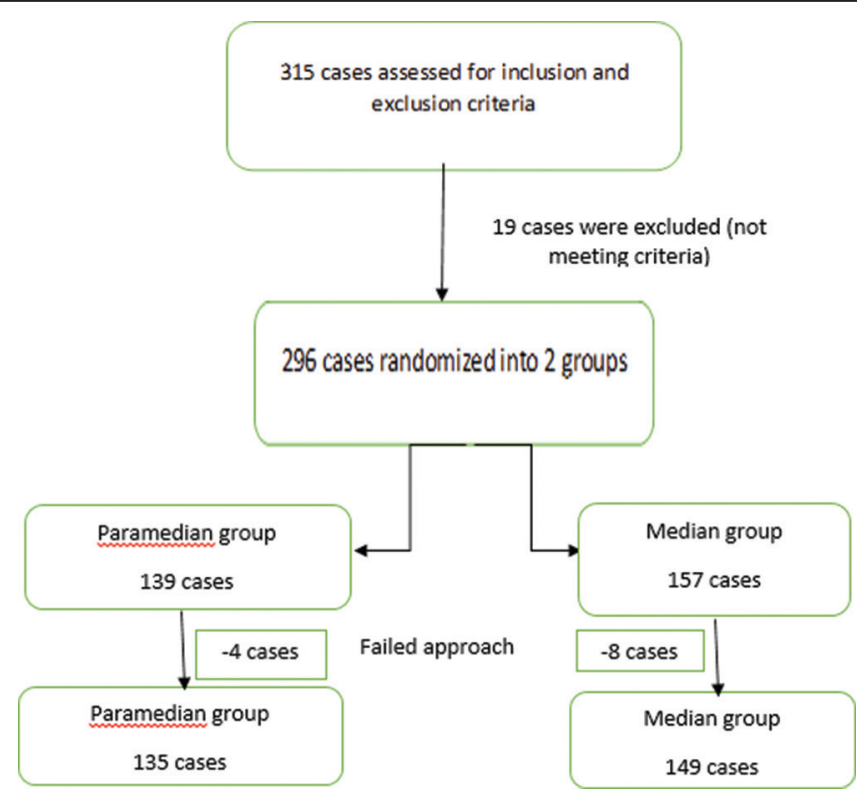

Figure 1: Study population flow diagram

a spinal needle at a $10-15^{\circ}$ cephalad angle at a point $1 \mathrm{~cm}$ lateral and $1 \mathrm{~cm}$ below the spinous process. Sharp needles G25 were used, and the needle's sharp blade cut parallel to the dural fibers for insertion. Twenty-five micrograms $(0.5 \mathrm{ml})$ of Fentanyl and $10 \mathrm{mg}(2 \mathrm{ml})$ of hyperbaric $0.5 \%$ bupivacaine were slowly injected after clear CSF flow was verified.

Any sensation paresthesia (unpleasant pain, burning, or electric sensation) observed by the anesthesiologist or reported by the patient on introduction of the Spinal needle is documented.

After receiving the spinal anesthesia, all the parturients were put in a 15 degree left lateral tilt position. After confirmation of an adequate level of sensory blockade, the surgeons were allowed to start the operation.

Hypotension and bradycardia were managed by ephedrine and atropine accordingly, and after the operation, all the patients were discharged to the postanesthesia care unit. The parturients were followed up postoperatively for any neurological complications after the wear off of the spinal anesthesia.

\section{Statistical analysis}

The sample size was calculated by using "ClinCalc" online sample size calculator software. The sample size was calculated according to the occurrence of paresthesia as the primary outcome according to a pilot study conducted before the original study. The incidence of paresthesia was $0 \%$ in the paramedian group and $12.5 \%$ in the median group. The calculated sample size was 154 patients (77 in each group) with alpha error 0.05 and $90 \%$ power of the study.

The Statistical Package for Social Science (SPSS 15) software was used for statistical analysis. 
The numeric variables were first tested for normality using Shapiro's test, then analyzed using unpaired $t$-test. The nominal data were analyzed using the Chi-square test or Mann-Whitney test as appropriate. $p<0.05$ was considered statistically significant.

\section{Results}

Regarding the demographic data, The mean age in the median group was 27.1 while in the paramedian group it was 30 with P-value $>0.001$. The weight was slightly higher in the paramedian group (mean $=83.8$ ) than the median group (mean $=79.6$ ) with a $p=0.017$. The height was slightly higher in the paramedian group (mean 161.5) than the median group (mean $=160)$ with P-value of 0.04 (Table 1).

Table 1: Demographic data of both groups. Data are expressed as mean (SD)

\begin{tabular}{llll}
\hline & Median & Paramedian & $\mathrm{p}$ value \\
& $\mathrm{n}=149$ & $\mathrm{n}=135$ & \\
\hline Age & $27.1(6)$ & $30(5.4)$ & $>0.001$ \\
weight & $79.6(14)$ & $83.8(15.7)$ & 0.017 \\
Height & $160(5.4)$ & $161.5(4.8)$ & 0.004 \\
\hline $\mathrm{p}<0.05$ is considered statistically significant. & &
\end{tabular}

The difference in the incidence of paresthesia was higher in the median group $(10.7 \%)$ than the paramedian group $(3.7 \%)$ with a statistical significance $(p=0.039)$. No postoperative neurological complications were reported in both groups (Table 2).

Table 2: Paresthesia in both groups. Data are expressed as number (percent)

\begin{tabular}{llll}
\hline & Median & Paramedian & p value \\
& $\mathrm{n}=149$ & $\mathrm{n}=135$ & \\
\hline Paresthesia & $16(10.7 \%)$ & $5(3.7 \%)$ & 0.039 \\
Neurological complications & 0 & 0 & \\
\hline $\mathrm{p}<0.05$ is considered statistically significant. & &
\end{tabular}

On recording the number of trials in each case of our study and testing its relation with the incidence of paresthesia, there was no statistically significant relation. The incidence of paraesthesia in the patients who required one, two, or three trials was comparable (7.1\% in one trial group, $7.4 \%$ in two trials group, and $25 \%$ in three trials group) (Table 3 ).

Table 3: Relationship between the number of trials and incidence of paresthesia. Data are expressed as number (percent)

\begin{tabular}{lllll}
\hline & 1 trial & 2 trials & 3 trials & $p$ value \\
$n=252$ & $n=27$ & $n=4$ & \\
\hline Paresthesia & $18(7.1)$ & $2(7.4)$ & $1(25)$ & 0.6 \\
\hline$p<0.05$ is considered statistically significant. The calculated percent is the percent of occurrence of
\end{tabular}
paresthesia in the cases sharing the same number of trials.

\section{Discussion}

The most serious complications with spinal anesthesia are neurological complications. It is difficult to estimate the incidence of neurological complications as they are relatively rare. Most of the reported neurological complications were preceded by paresthesia during the introduction of the spinal anesthesia needle.

Our results showed that the paramedian group had slightly higher age, weight, and height with a statistically significant difference $(p=0.017)$.

The success rate was $97.1 \%$ for the paramedian group, compared to $94.9 \%$ for the median group. The success rate was not $100 \%$ because we did not attempt more than three trials for each technique and shift to another technique after the third trial to avoid exhausting the patients. Yet, the paramedian group had a higher rate of success. The significance of this success shows more when we put into consideration that the paramedian group had a higher weight.

We found that the incidence of paresthesia the spinal anesthesia is significantly higher in the median group than in the paramedian group with a $p=0.039$. The importance of this finding is that the paramedian technique is probably safer regarding the incidence of neurologic complications with spinal anesthesia. All the patients were monitored during the anesthesia intake, intraoperatively, and for $24 \mathrm{~h}$ postoperatively for the occurrence of neurological complications (other than paresthesia) and both groups didn't show any neurological complications.

As far as our knowledge no studies were done to assess the incidence of paresthesia with different approaches (median and paramedian approaches) to spinal anesthesia in cesarean sections. One study that was originally comparing the safety and effectiveness of median and paramedian approaches in 100 parturients found that paresthesia was higher in the median group. The results were statistically non-significant where the $\mathrm{p}=0.37[10]$

Our results were consistent with the results of Singh et al., who compared the two approaches in elderly patients above fifty, and found that $10 \%$ of the median approach group suffered from paraesthesia, compared to only $4 \%$ in the paramedian group [11].

In contradiction to our findings, another study originally comparing the difference in the incidence of post-spinal back pain between midline and paramedian approaches for spinal anesthesia found that the incidence of paresthesia was higher in the paramedian group than in the median group. The $p=0.59$ and that was considered statistically non-significant [12].

Regarding the analysis of the incidence of paraesthesia in relation to the number of trials, the patients who required one or two trials had a comparable rate of paraesthesia $(7.1 \%$ in one trial group and $7.4 \%$ in two trials group). The true difference was between these two groups and the three trials group, where one of the four patients in this group $(25 \%)$ had paraesthesia. Since the number in the three trials group was very small, the results were statistically 
insignificant, and a correlation between the number of trials and the occurrence of paraesthesia could not be calculated. Yet, this finding is consistent with the findings of Blomberg et al. They found a statistically significant difference between the two techniques regarding the repeated number of attempts and the incidence of paraesthesia Their study was done on 49 patients that received lumbar epidural anesthesia for transurethral resection of the prostate [13].

This study included a relatively large number of patients of a homogenous group (parturient females between the age of 18 and 40). All patients underwent the same surgery. We found that the paramedian approach was more successful and much safer than the median approach. Further studies with a larger number of patients might be required to compare both approaches as regard more rare complications, especially neurological ones. Furthermore, they are needed to find the correlation between the number of attempts, and the occurrence of different complications.

\section{References}

1. World Health Organization. Caesarean Section Rates. Geneva: World Health Organization; 2015. p. 8.

2. Miseljic N, Basic E, Miseljic S. Causes of an increased rate of caesarean section. Mater Sociomed. 2018;30(4):287-9. https:// doi.org/10.5455/msm.2018.30.287-289

PMid:30936794

3. van Erp M, Ortner C, Jochberger S, Klein KU. Recent standards in management of obstetric anesthesia. Wien Med Wochenschr 2017;167:374-89. https://doi.org/10.1007/s10354-017-0584-0 PMid:28744777

4. Boon JM, Prinsloo E, Raath RP. A paramedian approach for epidural block: An anatomic and radiologic description. Reg Anesth Pain Med. 2003;28(3):221-7. https://doi. org/10.1097/00115550-200305000-00009

PMid: 12772140

5. Freshour CR, Constance D. ASRA practice advisory on neurologic complications in regional anesthesia and pain medicine. NIH Public Access. 2018;33(5):404-15.

PMid:18774509

6. Emami SA, Sahebkar A, Javadi B. Paresthesia: A review of its definition, etiology and treatments in view of the traditional medicine. Curr Pharm Des. 2015;22(3):321-7. https://doi. org/10.2174/1381612822666151112145348 PMid:26561077

7. Imbelloni LE, Gouveia MA. Paresthesia in spinal anesthesia. In: Imbelloni LE, Gouveia MA, editors. Paresthesia. Rijeka: IntechOpen; 2012. https://doi.org/10.5772/1567

8. Esther B, Hans-Fritz G, Marcus MA. Paraesthesia in Regional Anaesthesia. Paresthesia. Berlin, Germany: ResearchGate; 2012. https://doi.org/10.5772/35541

9. Imbelloni LE, Pitombo PF, Ganem EM. The incidence of paresthesia and neurologic complications after lower spinal thoracic puncture with cut needle compared to pencil point needle. Study in 300 patients. J Anesthe Clinic Res. 2010;1:106. http://doi.org/10.4172/2155-6148.1000106.

10. Kanagarajan $M$, Vanishree $C$, Jeeva G. Median and paramedian approach for spinal anaesthesia for caesarean delivery: A comparative analysis of safety and effectiveness. Indian J Clin Anaesth. 2017;4(4):518-22.

11. Singh P, Agrawal S, Dwivedi S, Yadav A. A comparative evaluation between median and paramedian approaches for sub-arachnoid block in elderly patients. Int J Res Med Sci. 2016;4(6):2069-72. https://doi.org/10.18203/2320-6012. ijrms20161762

12. Dadkhah $P$, Hashemi M, Gharaei B, Bigdeli MH, Solhpour A Comparison of Post-spinal Back Pain After Midline Versus Paramedian Approaches for Urologic Surgeries. Available from: https://asja.springeropen.com/articles/10.1186/s42077020-00088-5. [Last accessed on 2021 Apr 22]. https://doi. org/10.1186/s42077-020-00088-5

13. Blomberg RG, Jaanivald A, Walther $S$. Advantages of the paramedian approach for lumbar epidural analgesia with catheter technique: A clinical comparison between midline and paramedian approaches. Anaesthesia. 1989;44(9):742-6. https://doi.org/10.1111/j.1365-2044.1989.tb09260.x

PMid:2478045 